UVX 2012, 01005 (2013)

DOI: $10.1051 /$ uvx/201301005

(C) Owned by the authors, published by EDP Sciences, 2013

\title{
Dynamique ultra-rapide de la transition de phase solide-liquide-vapeur par spectroscopie XANES résolue en temps
}

\author{
P.M. Leguay ${ }^{1}$, a , B. Chimier ${ }^{1}$, P. Combis ${ }^{3}$, F. Deneuville ${ }^{1}$, D. Descamps ${ }^{1}$, \\ C. Fourment ${ }^{1}$, C. Goyon ${ }^{1}$, M. Harmand ${ }^{1}$, S. Hulin ${ }^{1}$, A. Lévy ${ }^{2}$, S. Petit ${ }^{1}$, \\ O. Peyrusse ${ }^{1}$, P. Renaudin ${ }^{3}$, J.J. Santos ${ }^{1}$ et F. Dorchies ${ }^{1}$ \\ 1 Univ. Bordeaux, CNRS, CEA, CELIA (Centre Lasers Intenses et Applications), UMR 5107, \\ 33400 Talence, France \\ 2 École Polytechnique, LULI (Laboratoire d'Utilisation des Lasers Intenses), UMR 7605, \\ 91128 Palaiseau, France \\ ${ }^{3}$ CEA-DAM-DIF, 91297 Arpajon, France
}

\begin{abstract}
Résumé. La spectroscopie d'absorption X près des seuils (XANES) est un outil puissant pour sonder la structure atomique (ordre local) et électronique (électrons de valence) dans de nombreux types de milieux, allant des molécules jusqu'à la matière condensée. À partir d'une source X laser-plasma ultra-brève compacte, nous avons réalisé des expériences de XANES avec une résolution temporelle ( 3 picosecondes) permettant de sonder, à l'échelle atomique, l'évolution d'une feuille d'aluminium illuminée par un laser ultra-bref. Les spectres d'absorption X révèlent, à haut flux, une transition ultra-rapide du solide cristallin au liquide désordonné, suivie par une relocalisation progressive des électrons de valence vers des orbitales atomiques (transition métal/non-métal et solide/vapeur). À plus bas flux, la matière reste dans une phase partiellement ordonnée aux temps longs, ce qui permet de suivre la perte progressive de l'ordre local et d'en estimer la durée à $5,7 \pm 3$ ps.
\end{abstract}

\section{INTRODUCTION}

Le développement rapide des systèmes lasers femtosecondes compacts a permis de nombreuses applications dans l'industrie et la médecine. Une impulsion laser focalisée peut déposer son énergie sur une surface micrométrique, permettant une ablation contrôlée de la matière, exploitable pour le micro-usinage ou pour réaliser des dépôts fins sur d'autres surfaces. Pendant l'ablation, la matière subit des transitions de phase ultra-rapides. Il est primordial de les comprendre et de déterminer leurs échelles de temps afin d'optimiser les procédés. D'un point de vue plus général, les lasers femtoseconde offrent une opportunité unique d'étudier ces transitions de phase à l'échelle de temps ultra-brève qui leur est naturelle.

Soumis à des flux de chauffage élevés, la matière atteint des états transitoires extrêmes, avec des pressions pouvant dépasser la limite statique accessible (quelques Mbar). L'étude de cette matière tiède et dense (WDM pour Warm Dense Matter) est un des grands défis de la physique contemporaine [1]. Les lasers femtosecondes peuvent chauffer la matière très rapidement, ce qui conduit à des transitions ultra-rapides solide-liquide-WDM suivies, à l'échelle picoseconde, par une expansion multiphase plus complexe. L'énergie laser est initialement déposée dans les électrons. Le taux de transfert énergétique électrons-ions étant limité, la matière traverse des états hautement hors équilibre.

a e-mail : leguay@celia.u-bordeaux1.fr

This is an Open Access article distributed under the terms of the Creative Commons Attribution License 2.0, which permits unrestricted use, distribution, and reproduction in any medium, provided the original work is properly cited. 


\section{Web of Conferences}

Afin de comprendre la dynamique d'interaction entre les électrons et les ions, l'enjeu expérimental est de combiner des mesures de leurs structures à l'échelle atomique, avec une résolution temporelle ultra-brève.

Récemment, de nouvelles techniques de diffraction ultra-rapide d'électrons [2,3] et de rayons $\mathrm{X}$ [4,5] ont été développées [6]. Elles ont permis de sonder avec succès la dynamique des phonons [7], les phénomènes de fusion [8,9] ou le renforcement de liaison dans la transition solide-WDM [10]. Mais elles ne sont plus adaptées dès que l'ordre à grande distance disparaît (liquide, WDM, vapeur). Dans ces situations, la spectroscopie d'absorption X près des seuils (XANES pour X-ray Absorption NearEdge Spectroscopy) est particulièrement appropriée [11], puisqu'elle sonde d'une part l'ordre à courte distance, qui persiste après la fusion, et d'autre part la densité des états électroniques inoccupés, ce qui permet d'accéder à la dynamique de l'interaction entre les électrons de valence et l'arrangement atomique.

Contrairement à la diffraction ultra-rapide, qui a été réalisée sur des lasers compacts, abordables et polyvalents, le développement des expériences compactes d'absorption X ultra-rapide a été limité par le très faible flux $X$ produit dans les spectres continus de Bremsstrahlung [12,13]. La première résolution de quelques ps a été obtenue en observant la disparition d'un pic d'absorption au moment de la photodissociation d'une molécule [14]. Les premières expériences de XANES ultra-rapide ont été réalisées récemment sur des installations de type synchrotron : en couplant une caméra à balayage de fente, d'une résolution de quelques picosecondes, avec un synchrotron [15] ; ou en utilisant le découpage («slicing»), pour atteindre une résolution sub-picoseconde [16,17]. L'utilisation de ces deux méthodes, pour des niveaux d'excitation élevés (où l'échantillon doit être renouvelé après chaque tir laser), est cependant limitée par le très faible nombre de photons disponibles par tir.

Afin de surmonter ces limitations, nous avons développé une source X compacte et brillante, adaptée aux expériences XANES [18], en optimisant l'émission thermique large bande de couche M de plasmas produits par laser dans le domaine multi-keV [19]. Le nombre de photons est de plusieurs ordres de grandeur supérieur au Bremsstrahlung utilisé précédemment [12,13]. Le caractère isotrope de cette source X quasi ponctuelle permet l'utilisation d'un dispositif dispersif d'absorption X, et donc d'obtenir un spectre complet à chaque tir. Ainsi, en utilisant un laser de $100 \mathrm{~mJ}$ travaillant à un taux de répétition de $10 \mathrm{~Hz}$, seuls quelques dizaines de tirs sont requis pour acquérir des spectres XANES exploitables, comme ceux présentés dans cet article. Ceci constitue une avancée importante pour la spectroscopie d'absorption X ultra-rapide.

\section{DISPOSITIF EXPÉRIMENTAL}

Avec cette source X compacte, nous avons réalisé l'étude résolue en temps du spectre XANES, autour du flanc K, au travers d'un échantillon d'aluminium ablaté par une impulsion laser femtoseconde. Le schéma expérimental est présenté sur la figure 1. Le faisceau laser est séparé en deux impulsions synchronisées. La première chauffe en 120 fs l'échantillon de $100 \mathrm{~nm}$ d'Al sur une zone ellipsoïdale de $700 \mu \mathrm{m} \times 350 \mu \mathrm{m}$. Deux expériences ont été réalisées dans ces conditions. Dans la première, la fluence de chauffage $\left(6 \mathrm{~J} / \mathrm{cm}^{2}\right)$ est plus d'un ordre de grandeur supérieure au seuil d'ablation [20], ce qui permet d'éviter la nucléation (régime de coexistence liquide-vapeur) dans les premières dizaines de picosecondes de l'expansion [21]. Dans cette situation, comme nous le montrerons plus loin, l'ordre local est très vite détruit, bien avant que l'équilibration se fasse entre électrons et ions. Dans la deuxième expérience, la fluence a été réduite $\left(0,3 \mathrm{~J} / \mathrm{cm}^{2}\right)$ de façon à maintenir, après l'équilibration électrons-ions, un ordre local partiel. Ceci permet d'évaluer le temps sur lequel ce dernier diminue.

La deuxième impulsion est focalisée sur une cible de conversion $\mathrm{X}$ (holmium), dans une tache focale de $80 \mu \mathrm{m}$, afin de produire la source $\mathrm{X}$ isotrope. Les photons sont collectés et dispersés par deux cristaux de Bragg sur une caméra CCD (Charge Coupled Device), afin d'enregistrer respectivement le spectre de référence de la source $\mathrm{X}$, et le spectre transmis à travers l'échantillon chauffé. Les détails concernant le spectromètre et la procédure d'extraction des spectres sont reportés dans la référence [22]. 


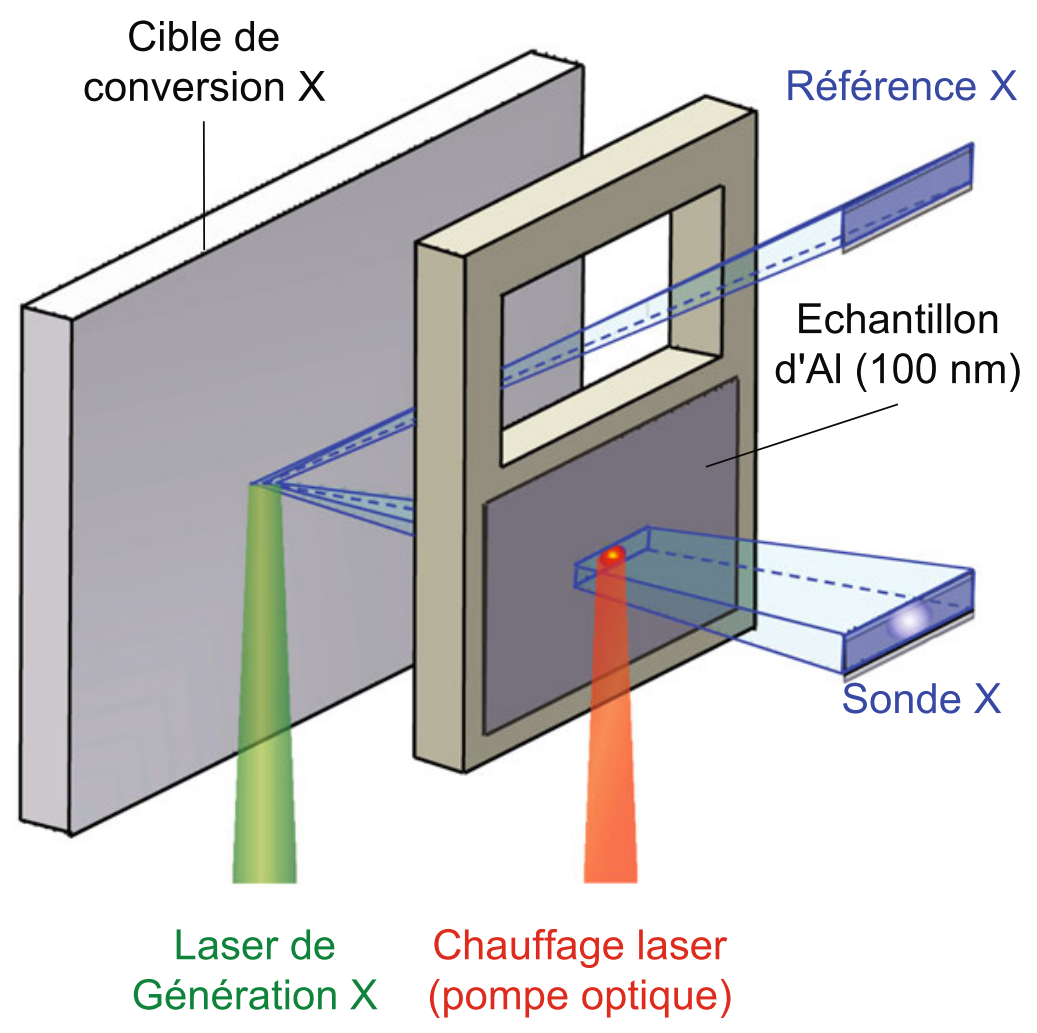

Figure 1. Schéma du montage d'absorption $X$ résolue en temps. La première impulsion laser (pompe optique) chauffe l'échantillon de $100 \mathrm{~nm}$ d'aluminium déposé sur une grille adaptée. La deuxième impulsion est focalisée sur une cible de conversion X qui, placée $7 \mathrm{~mm}$ derrière l'échantillon, génère une émission X brillante, large-bande, isotrope et ultra-brève. Le rayonnement $\mathrm{X}$ est dispersé spectralement par deux cristaux de Bragg (non représentés) sur une caméra CCD. Le faisceau du haut mesure directement le spectre X émis par la source (référence), alors que celui du bas mesure le spectre transmis à travers l'échantillon d'aluminium chauffé (sonde). Les angles solides de collection sont représentés sur la figure. Le retard entre les deux impulsions laser est modifié afin d'obtenir le spectre XANES résolu en temps par rapport au chauffage.

La résolution spatiale du dispositif est de $\sim 100 \mu$ m suivant l'axe horizontal de la figure 1, ce qui permet d'aligner précisément les zones chauffée et sondée. Le délai entre la pompe optique et la sonde X est contrôlé par une ligne à retard sans gigue temporelle. La résolution temporelle est principalement limitée par la durée de la sonde $\mathrm{X}$, évaluée à $3,15 \pm 0,25$ ps rms avec une caméra à balayage de fente.

\section{TRANSITION SOLIDE-LIQUIDE-VAPEUR À HAUT FLUX}

Les spectres XANES résolus en temps lors du chauffage à haut flux sont présentés dans la figure 2. Chaque spectre est obtenu en intégrant le rayonnement $\mathrm{X}$ collecté dans une zone rectangulaire de l'échantillon de $450 \mu \mathrm{m} \times 140 \mu \mathrm{m}$, dans laquelle le chauffage est homogène dans une barre d'erreur de $20 \%$. Le premier spectre, obtenu 7,5 ps avant la pompe optique, est celui de l'aluminium à température ambiante. Il présente un flanc $\mathrm{K}$ raide, ce qui révèle une nette séparation entre les états occupés et vacants dans la bande de conduction, à l'énergie de Fermi. Le flanc K est suivi des modulations XANES attendues pour la structure cubique à faces centrées de l'aluminium solide. Leur disparition 2,5 ps après le chauffage indique une perte de l'ordre à courte portée, ce qui témoigne a fortiori 


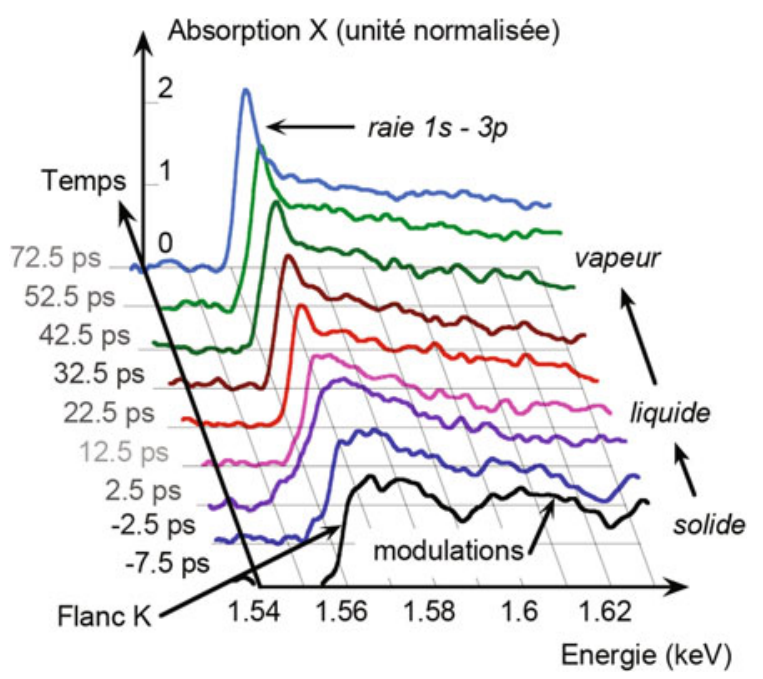

Figure 2. Spectres XANES mesurés à haut flux $\left(6 \mathrm{~J} / \mathrm{cm}^{2}\right)$, en fonction du retard entre la sonde $\mathrm{X}$ et la pompe optique. L'origine est fixée quand le maximum des deux impulsions coïncide. Le spectre collecté au temps $-7,5 \mathrm{ps}$ présente les motifs attendus pour l'aluminium non chauffé : un flanc K raide, suivi par des modulations XANES. Ces modulations disparaissent très rapidement, révélant une perte rapide de l'ordre atomique à courte portée (par conséquent une transition solide-liquide ultra-rapide). À $2,5 \mathrm{ps}$, la pente du flanc est réduite par l'augmentation de la température électronique. Aux temps plus longs, on observe une transition, du flanc $\mathrm{K}$ à la raie d'absorption atomique $1 s-3 p$, ce qui indique la relocalisation progressive des électrons de valence dans les orbitales atomiques pendant la transition liquide-vapeur.

d'une transition solide-liquide ultra-rapide. Au même moment, l'adoucissement de la pente du flanc K confirme l'élargissement thermique du niveau de Fermi. L'interpolation de cette courbe avec la distribution de Fermi-Dirac [23] permet d'estimer une température électronique de $1,8 \pm 0,5 \mathrm{eV}$. Après $\sim 20 \mathrm{ps}$, les spectres indiquent une transition progressive depuis le flanc $\mathrm{K}$ vers la raie atomique $1 s-3 p$.

Nous avons utilisé un code hydrodynamique à deux températures, ESTHER [20], pour déterminer l'évolution macroscopique de l'échantillon chauffé. Comme l'épaisseur de l'échantillon est supérieure à l'épaisseur de peau du laser, de forts gradients longitudinaux de température sont présents au départ. Ils sont lissés en quelques picosecondes par la conduction thermique. Les valeurs des températures électronique $T_{e}$ et ionique $T_{i}$, et de la densité $N_{i}$, moyennées sur l'épaisseur de l'échantillon, sont reportées dans la figure 3 aux instants des mesures XANES. Les barres d'erreur représentent les inhomogénéités (écart type suivant le profil longitudinal). L'énergie laser est d'abord déposée sur les électrons (pendant la durée de la pompe : $120 \mathrm{fs}$ ). Le transfert d'énergie des électrons au réseau suit jusqu'à l'équilibration, qui est atteinte après $\sim 10 \mathrm{ps}$. Le haut niveau d'excitation électronique impose à $T_{i}$ de dépasser la température de fusion $(0,08 \mathrm{eV})$ très rapidement. La transition solideliquide a donc lieu sur une échelle de temps sub-picoseconde. Comme la phase liquide conserve un ordre à courte portée, les modulations XANES persistent, puis disparaissent avec l'augmentation de $T_{i}$. Expérimentalement, les modulations XANES ont disparu 2,5 ps après le chauffage, alors que $T_{i}$ est estimé à $0,65 \pm 0,25 \mathrm{eV}$, ce qui est en accord avec des calculs publiés sur le chauffage isochore par protons [23], où la disparition des modulations XANES est observée pour des températures comprises entre 0,5 et $1 \mathrm{eV}$ à l'équilibre thermique. Au même moment (2,5 ps), $T_{e}$ est estimée à $2,5 \pm 0,7 \mathrm{eV}$, ce qui est en accord avec la valeur de $1,8 \pm 0,5 \mathrm{eV}$ déduite de l'élargissement du flanc $\mathrm{K}$ d'absorption.

Le calcul reporté en figure 3 montre que l'expansion a lieu sur la même échelle de temps que l'équilibration électrons-ions. L'échantillon reste en dessous du point critique liquide-gaz [24], ce 


\section{UVX 2012}

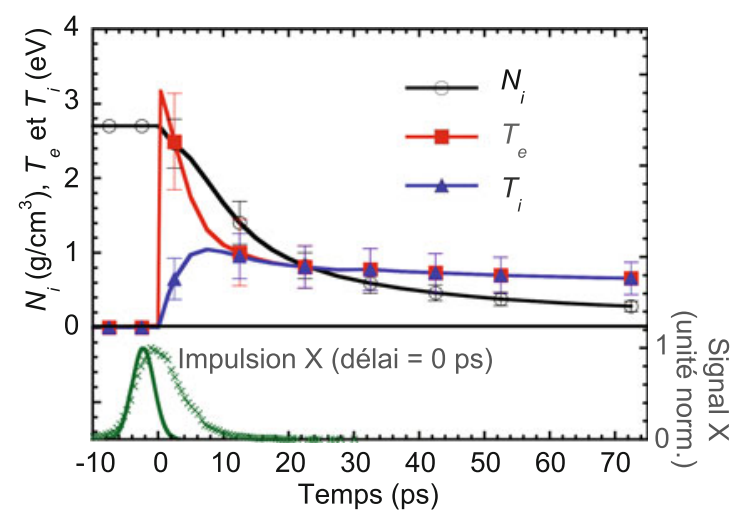

Figure 3. Profils temporels de la densité et des températures de l'aluminium, calculés avec un code hydrodynamique à deux températures. L'échantillon de $100 \mathrm{~nm}$ d'aluminium est chauffé par une impulsion laser de $6 \mathrm{~J} / \mathrm{cm}^{2}$, de $120 \mathrm{fs}$, à $60^{\circ}$ d'incidence et en polarisation $p$. Haut : densité $N_{i}$, températures électronique $T_{e}$ et ionique $T_{i}$ en fonction du temps (courbes). Afin de comparer aux résultats expérimentaux de la figure 2 , les valeurs calculées aux temps de mesure XANES sont représentées par des symboles ( $N_{i}$ cercles, $T_{e}$ carrés, $T_{i}$ triangles). Bas : profil temporel de la source $\mathrm{X}$ (croix) pour un retard de 0 ps entre les deux impulsions, comparé à la réponse temporelle de la caméra à balayage de fente (trait). Après déconvolution, la durée de la source X est de $3,15 \pm 0,25 \mathrm{ps} \mathrm{rms.}$

qui permet d'éviter toute nucléation. Dans ces conditions, l'augmentation de la distance moyenne entre atomes voisins est directement donnée par la diminution de la densité due à l'expansion hydrodynamique. La transition progressive, observée dans la figure 2, entre le flanc $\mathrm{K}$ et la raie d'absorption atomique $1 s-3 p$, commence environ $20 \mathrm{ps}$ après le chauffage, c'est-à-dire quand la densité ionique atteint le tiers de la densité solide $\left(\sim 1 \mathrm{~g} / \mathrm{cm}^{3}\right)$. À ce délai, la distance moyenne entre les atomes a augmenté de $\sim 40 \%$ par rapport au cas solide. Les spectres d'absorption X sont en bon accord avec une expérience et des simulations $a b$ initio de dynamique moléculaire quantique, réalisées à l'équilibre thermique [26], la transition flanc $\mathrm{K} /$ raie $1 s-3 p$ étant attendue quand la densité passe sous $1 \mathrm{~g} / \mathrm{cm}^{3}$.

\section{DYNAMIQUE DE LA DIMINUTION DE L'ORDRE LOCAL À BAS FLUX}

Lors de l'absorption d'un photon X par un atome, la fonction d'onde du photoélectron créé se diffuse sur les atomes voisins. L'interférence entre les multiples diffusions modifie le spectre dans la zone d'énergie au delà du flanc. Ainsi, les modulations observées révèlent l'ordre local autour de l'atome absorbant. L'étude reportée dans la référence [25] a montré que la zone spectrale comprise entre 1,555 et 1,57 keV est sensible à la densité de l'échantillon : la raie d'absorption observée sur la figure 2 apparait dès que la densité est divisée par 2 ou 3 par rapport à celle du solide. Une étude du chauffage isochore de l'aluminium à l'équilibre thermique [23] a montré que le spectre XANES est modifié par la température entre 1,58 et 1,62 keV. L'amplitude de la bosse observée diminue quand la température augmente ; elle disparait pour une température critique entre 0,5 et $1 \mathrm{eV}$, ce qui traduit la perte de l'ordre local dans le matériau.

Dans les spectres mesurés à haut flux (cf. figure 2), cette modulation spectrale disparaitt en un temps plus court que la résolution temporelle, ce qui indique que la température ionique a rapidement dépassé la température critique. Afin de pouvoir suivre plus finement la perte d'ordre local, nous avons réalisé une deuxième expérience, basée sur le même dispositif expérimental (mesure de spectres XANES résolus en temps après chauffage optique d'un échantillon d'aluminium), avec un flux laser plus bas $\left(0,3 \mathrm{~J} / \mathrm{cm}^{2}\right)$. Les données sont en cours d'analyse et feront l'objet d'une prochaine publication, mais les premiers résultats sont prometteurs : on observe une perte progressive de la modulation précédemment 


\section{Web of Conferences}

décrite (bosse du spectre entre 1,58 et 1,62 keV), mais elle persiste et se stabilise aux temps longs. Par conséquent, l'équilibration électrons-ions s'est faite en dessous de la température critique. Les données permettent donc de suivre la dynamique de la diminution de l'ordre local dans le matériau, et d'en déterminer un temps caractéristique de $5,7 \pm 3 \mathrm{ps}$.

Afin de valider ces résultats, l'expérience a été simulée à l'aide du même code hydrodynamique à deux températures. Le dépôt énergétique se fait sur les électrons dans l'épaisseur de peau. Par conduction thermique, le chauffage est homogénéisé sur l'épaisseur de l'échantillon en moins de 2 ps. Le passage de l'état solide à l'état liquide se fait sur la même échelle de temps, puis la matière reste majoritairement à l'état liquide aux temps longs (de $\sim 10$ à 100 ps). Aux temps plus courts, l'hydrodynamique est complexe. Une couche de liquide se développe peu à peu sur les deux faces de la feuille. La densité $\mathrm{du}$ liquide $\left(\sim 2,3 \mathrm{~g} / \mathrm{cm}^{3}\right)$ étant plus faible que celle du solide $\left(\sim 2,7 \mathrm{~g} / \mathrm{cm}^{3}\right)$, ceci se traduit par une détente progressive de la feuille. Les deux fronts de détente (frontières liquide-solide) se rejoignent au milieu de la cible au bout d'une dizaine de picosecondes.

Dans des études précédentes sur la dynamique ultra-rapide d'un échantillon mince soumis à l'ablation laser [2,27], les calculs ont été basés sur l'hypothèse que l'équilibration électrons-ions se déroule à densité constante et homogène, puisque la détente hydrodynamique a lieu sur des temps plus longs. La dilatation progressive du matériau, de la densité du solide à celle du liquide, observée ici dès les premiers instants, interroge la validité de cette hypothèse d'isochoricité.

\section{CONCLUSIONS ET PERSPECTIVES}

Les mesures de XANES résolues en temps fournissent des données, à l'échelle atomique, sur la transition ultra-rapide du solide vers le liquide puis la vapeur, lors de l'ablation d'une feuille mince d'aluminium par une impulsion laser femtoseconde. Ce régime correspond à la partie basse température de la matière tiède et dense (quelques eV). Contrairement au signal de diffraction, qui disparaît dès que l'ordre cristallin est rompu, les spectres XANES fournissent des informations structurelles sur les atomes et les électrons, au delà de la fusion. Dans la phase solide initiale, les atomes sont ordonnés dans un réseau cristallin. Les électrons de valence sont délocalisés dans la bande de conduction de l'aluminium. Ils sont rapidement chauffés par l'impulsion laser, ce qui élargit dans un premier temps le facteur d'occupation de Fermi-Dirac. Le transfert d'énergie électron-ion conduit à une transition du solide froid cristallin vers le liquide métallique désordonné, puis à une diminution de l'ordre atomique à courte portée. Deux expériences ont été menées, dans deux régimes différents de flux laser.

À haut flux $\left(6 \mathrm{~J} / \mathrm{cm}^{2}\right)$, les modulations XANES révélant l'ordre local disparaissent en moins de 2,5 ps. La transition solide-liquide a donc lieu sur un temps plus court. Les calculs suggèrent que la température ionique dépasse la température de fusion en moins de 1 ps, à cause du haut niveau d'excitation. Ce temps de transition est court comparé au temps de 3,5 ps obtenu par des mesures récentes de diffraction électronique [2], ce qui est compréhensible puisque cette expérience a été réalisée à un flux plus faible, de $70 \mathrm{~mJ} / \mathrm{cm}^{2}$. Par la suite, la transition du liquide à la vapeur atomique apparaît au bout de $\sim 20 \mathrm{ps}$. Elle est révélée par la relocalisation progressive des électrons de valence depuis la bande de conduction (métal), jusqu'aux orbitales atomiques (non-métal), quand la distance moyenne entre atomes croît. L'accord entre les calculs et les résultats expérimentaux montre que cette échelle de temps est pilotée par l'expansion hydrodynamique, qui dépend du niveau de pression thermique atteint après le chauffage. Les mêmes calculs indiquent que l'équilibration électrons-ions se produit sur une échelle de temps $(\sim 10 \mathrm{ps})$ similaire à celle de l'expansion. Les mesures XANES résolues en temps ne permettent pas de la déterminer, puisque les modulations disparaissent bien avant l'équilibration (l'ordre local est trop diminué pour être mesurable par le XANES).

À bas flux $\left(0,3 \mathrm{~J} / \mathrm{cm}^{2}\right)$, l'expansion hydrodynamique, plus lente, ne devrait pas altérer l'équilibration électrons-ions. Les mesures montrent en effet que la feuille reste à une densité relativement proche de celle du solide $\left(>2 \mathrm{~g} / \mathrm{cm}^{3}\right)$, même aux temps longs $(\sim 100 \mathrm{ps})$. De plus, la température ionique après 


\section{UVX 2012}

équilibration étant suffisamment basse, les modulations XANES restent visibles. Ceci nous permet de déduire un temps caractéristique de diminution de l'ordre local de 5,7 \pm 2 ps.

En plus de l'intérêt fondamental de la dynamique de transitions de phase, cette expérience de XANES ultra-rapide a été réalisée avec un dispositif tout-optique compact. La résolution temporelle est intrinsèquement limitée par la durée de la source $X(3,15 \pm 0,25 \mathrm{ps} \mathrm{rms})$, mais pourrait atteindre le domaine sub-picoseconde en utilisant des cibles d'agrégats pour produire les rayons X [28], ou même le domaine femtoseconde en utilisant l'émission bêtatron, issue de l'interaction d'une impulsion laser relativiste avec un jet de gaz [29]. Le rayonnement X produit par les lasers à électrons libres, qui offre à la fois une résolution femtoseconde, et un nombre de photons par tirs important [30-32] est très prometteur. Cependant, même si des stations expérimentales sont réservées pour les études de la WDM, les coûts de fonctionnement en limitent nécessairement l'accès. À cet égard, le développement de dispositifs compacts d'absorption X ultra-rapide aura certainement un impact important dans l'exploration de nombreux systèmes transitoires, allant des réactions chimiques aux états extrêmes de la matières, ou à la physique hors équilibre.

\section{Remerciements}

Ce travail a bénéficié du soutien financier de l'Agence Nationale de la Recherche (OEDYP No ANR-09-BLAN0206-01) et du Conseil Régional d'Aquitaine (POLUX No 2010-13-04-002 et COLA2 No 2.1.3-09010502). Les auteurs remercient Rodrigue Bouillaud pour son support technique, et Capucine Medina pour son aide précieuse sur le laser.

\section{Références}

[1] National Research Council, Frontiers in High Energy Density Physics: The X-Games of Contemporary Science (National Academic Press, Washington, D.C., 2003)

[2] B.J. Siwick et al., Science 302, 1382 (2003)

[3] N. Gedik et al. Science 316, 425 (2007)

[4] C. Rischel et al., Nature 390, 490 (1997)

[5] A.M. Lindenberg et al., Science 308, 392 (2005)

[6] T. Pfeifer et al., Rev. Prog. Phys. 69, 443 (2006)

[7] K. Sokolowski-Tinten et al., Nature 422, 287 (2003)

[8] C.W. Siders et al., Science 286, 1340 (1999)

[9] A. Rousse et al., Nature 410, 65 (2001)

[10] R. Ernstorfer et al., Science 323, 1033 (2009)

[11] Ch. Bressler and M. Chergui, Chem. Rev. 104, 1781 (2004)

[12] T. Lee et al., J. Chem. Phys. 122, 084506 (2005)

[13] J. Chen et al., Chem. Phys. Lett. 437, 50 (2007)

[14] F. Ráksi et al., J. Chem. Phys. 104, 6066 (1996)

[15] S.L. Johnson et al., Phys. Rev. Lett. 91, 157403 (2003)

[16] A. Cavalleri et al., Phys. Rev. Lett. 95, 067405 (2005)

[17] Ch. Bressler et al., Science 323, 489 (2009)

[18] F. Dorchies et al., Appl. Phys. Lett. 93, 121113 (2008)

[19] M. Harmand et al., Phys. Plasmas 16, 063301 (2009)

[20] J.P. Colombier et al., Phys. Rev. B 71,165406 (2005)

[21] A.M. Lindenberg et al., Phys. Rev. Lett. 100, 135502 (2008)

[22] A. Lévy et al., Rev. Sc. Instrum. 81, 063107 (2010)

[23] A. Mancic et al., Phys. Rev. Lett. 104, 035002 (2010)

[24] B. Chimier et al., Phys. Rev. B 75, 195124 (2007)

[25] A. Lévy et al., Phys. Rev. Lett. 108, 055002 (2012) 


\section{Web of Conferences}

[26] V. Recoules and S. Mazevet, Phys. Rev. B 80, 064110 (2009)

[27] B.I. Cho et al., Phys. Rev. Lett. 106, 167601 (2011)

[28] F. Dorchies et al., Phys. Rev. Lett. 100, 205002 (2008)

[29] A. Rousse et al., Phys. Rev. Lett. 93, 135005 (2004)

[30] B.D. Paterson and R. Abela, Phys. Chem. Chem. Phys. 12, 5647 (2010)

[31] B. Nagler et al., Nature Phys. 5, 693 (2009)

[32] S.M. Vinko et al., Phys. Rev. Lett. 104, 225001 (2010) 